\title{
On the existence of solutions for a nonconvex hyperbolic differential inclusion of third order
}

\author{
Aurelian Cernea ${ }^{\otimes}$ \\ Appeared 11 August 2016 \\ Communicated by Tibor Krisztin
}

University of Bucharest, Academiei 14, Bucharest, RO-010014, Romania

\begin{abstract}
We establish some Filippov type existence theorems for solutions of a Darboux problem associated to a third order hyperbolic differential inclusion.
\end{abstract}

Keywords: set-valued map, hyperbolic differential inclusion, decomposable set.

2010 Mathematics Subject Classification: 34A60, 35L30.

\section{Introduction}

In this paper we study the following Darboux problem for a third order hyperbolic differential inclusion

$$
u_{x y z}(x, y, z) \in F(x, y, z, u(x, y, z)), \quad(x, y, z) \in \Pi:=\left[0, T_{1}\right] \times\left[0, T_{2}\right] \times\left[0, T_{3}\right]
$$

with the initial values

$$
\begin{aligned}
u(x, y, 0) & =\varphi(x, y), & & (x, y) \in \Pi_{1}:=\left[0, T_{1}\right] \times\left[0, T_{2}\right], \\
u(0, y, z) & =\psi(y, z), & & (y, z) \in \Pi_{2}:=\left[0, T_{2}\right] \times\left[0, T_{3}\right], \\
u(x, 0, z) c & =\chi(x, z), & & (x, z) \in \Pi_{3}:=\left[0, T_{1}\right] \times\left[0, T_{3}\right],
\end{aligned}
$$

where $\varphi, \psi, \chi$ are absolutely continuous functions satisfying

$$
\begin{array}{ll}
u(x, 0,0)=\varphi(x, 0)=\chi(x, 0)=: v_{1}(x), & x \in\left[0, T_{1}\right], \\
u(0, y, 0)=\varphi(0, y)=\psi(y, 0)=: v_{2}(y), & y \in\left[0, T_{2}\right], \\
u(0,0, z)=\psi(0, z)=\chi(0, z)=: v_{3}(z), & z \in\left[0, T_{3}\right], \\
u(0,0,0)=v_{1}(0)=v_{2}(0)=v_{3}(0)=: v_{0} &
\end{array}
$$

and $T_{1}, T_{2}, T_{3}>0$ and $F: \Pi \times \mathbb{R}^{n} \rightarrow \mathcal{P}\left(\mathbb{R}^{n}\right)$ is a set-valued map.

Qualitative properties, existence results and structure of the set of solutions of the Darboux problem (1.1)-(1.2) have been studied by many authors [4,7-9] etc.

\footnotetext{
${ }^{\bowtie}$ Email: acernea@fmi.unibuc.ro
} 
The aim of the present paper is twofold. On one hand, we show that Filippov's ideas [5] can be suitably adapted in order to obtain the existence of a solution of problem (1.1)-(1.3). We recall that for a first order differential inclusion defined by a Lipschitzian set-valued map with nonconvex values Filippov's theorem ([5]) consists in proving the existence of a solution starting from a given "almost" solution. Moreover, the result provides an estimate between the starting "quasi" solution and the solution of the differential inclusion. On the other hand, we prove the existence of solutions continuously depending on a parameter for problem (1.1)-(1.3). This result may be interpreted as a continuous variant of Filippov's theorem for problem (1.1)-(1.3). The key tool in the proof of this theorem is a result of Bressan and Colombo [2] concerning the existence of continuous selections of lower semicontinuous multifunctions with decomposable values. This result allows to obtain a continuous selection of the solution set of the problem considered.

In the literature there are several papers concerning the existence of solutions for higher order differential inclusions defined by Lipschitzian set-valued maps; we mention the paper [1] for a boundary value problem associated to a second-order differential inclusion which also uses Filippov's techniques.

Our results may be interpreted as extensions of previous results of Staicu [6] and Tuan $[10,11]$ obtained for "classical" hyperbolic differential inclusions.

The paper is organized as follows: in Section 2 we briefly recall some preliminary results that we will use in the sequel and in Section 3 we prove the main results of the paper.

\section{Preliminaries}

In this section we sum up some basic facts that we are going to use later.

Let $(X, d)$ be a metric space. The Pompeiu-Hausdorff distance of the closed subsets $A, B \subset X$ is defined by $d_{H}(A, B)=\max \left\{d^{*}(A, B), d^{*}(B, A)\right\}, d^{*}(A, B)=\sup \{d(a, B) ; a \in A\}$, where $d(x, B)=\inf \{d(x, y) ; y \in B\}$. With $\operatorname{cl}(A)$ we denote the closure of the set $A \subset X$.

Consider $I_{1}=\left[0, T_{1}\right], I_{2}=\left[0, T_{2}\right], I_{3}=\left[0, T_{3}\right]$ and $\Pi=\left[0, T_{1}\right] \times\left[0, T_{2}\right] \times\left[0, T_{3}\right]$. Denote by $\mathcal{L}(\Pi)$ the $\sigma$-algebra of the Lebesgue measurable subsets of $\Pi$ and by $\mathcal{B}\left(\mathbb{R}^{n}\right)$ the family of all Borel subsets of $\mathbb{R}^{n}$.

Let $C\left(\Pi, \mathbb{R}^{n}\right)$ be the Banach space of all continuous functions from $\Pi$ to $\mathbb{R}^{n}$ with the norm $\|u\|_{C}=\sup \{\|u(x, y, z)\| ;(x, y, z) \in \Pi\}$ where $\|\cdot\|$ is the Euclidean norm on $\mathbb{R}^{n}$, and $L^{1}\left(\Pi, \mathbb{R}^{n}\right)$ be the Banach space of integrable functions $u: \Pi \rightarrow \mathbb{R}^{n}$ with the norm $\|u\|_{1}=$ $\int_{0}^{T_{1}} \int_{0}^{T_{2}} \int_{0}^{T_{3}}\|u(x, y, z)\| d x d y d z$.

Recall that a subset $D \subset L^{1}\left(\Pi, \mathbb{R}^{n}\right)$ is said to be decomposable if for any $u(\cdot), v(\cdot) \in D$ and any subset $A \in \mathcal{L}(\Pi)$ one has $u \chi_{A}+v \chi_{B} \in D$, where $B=I \backslash A$. We denote by $\mathcal{D}$ the family of all decomposable closed subsets of $L^{1}\left(\Pi, \mathbb{R}^{n}\right)$.

Let $F(\cdot, \cdot): \Pi \times \mathbb{R}^{n} \rightarrow \mathcal{P}\left(\mathbb{R}^{n}\right)$ be a set-valued map. Recall that $F(\cdot, \cdot)$ is called $\mathcal{L}(\Pi) \otimes$ $\mathcal{B}\left(\mathbb{R}^{n}\right)$ measurable if for any closed subset $C \subset \mathbb{R}^{n}$ we have $\left\{(x, y, z, u) \in \Pi \times \mathbb{R}^{n} ; F(x, y, z, u) \cap\right.$ $C\} \neq \varnothing\} \in \mathcal{L}(\Pi) \otimes \mathcal{B}\left(\mathbb{R}^{n}\right)$.

We recall now some results that we are going to use in the next section. The next lemma may be found in [3].

Lemma 2.1. Let $H: \Pi \rightarrow \mathcal{P}\left(\mathbb{R}^{n}\right)$ be a compact valued measurable multifunction and $v: \Pi \rightarrow \mathbb{R}^{n}$ a measurable function.

Then there exists a measurable selection $h$ of $H$ such that

$$
\|v(x, y, z)-h(x, y, z)\|=d(v(x, y, z), H(x, y, z)), \quad \text { a.e. }(\Pi) \text {. }
$$


Next $(S, \mathrm{~d})$ is a separable metric space and $X$ is a Banach space. We recall that a multifunction $G(\cdot): S \rightarrow \mathcal{P}(X)$ is said to be lower semicontinuous (1.s.c.) if for any closed subset $C \subset X$, the subset $\{s \in S ; G(s) \subset C\}$ is closed in $S$.

The proofs of the following two lemmas are in [6].

Lemma 2.2. Let $F^{*}(\cdot, \cdot): \Pi \times S \rightarrow \mathcal{P}\left(\mathbb{R}^{n}\right)$ be a closed valued $\mathcal{L}(\Pi) \otimes \mathcal{B}(S)$ measurable multifunction such that $F^{*}((x, y, z), \cdot)$ is l.s.c. for any $(x, y, z) \in \Pi$.

Then the set-valued map $G(\cdot)$ defined by

$$
G(s)=\left\{v \in L^{1}\left(\Pi, \mathbb{R}^{n}\right) ; v(x, y, z) \in F^{*}(x, y, z, s) \text { a.e. }(\Pi)\right\}
$$

is l.s.c. with nonempty decomposable closed values if and only if there exists a continuous mapping $p(\cdot): S \rightarrow L^{1}\left(\Pi, \mathbb{R}^{n}\right)$ such that

$$
d\left(0, F^{*}(x, y, z, s)\right) \leq p(s)(x, y, z) \text { a.e. }(\Pi), \forall s \in S .
$$

Lemma 2.3. Let $G(\cdot): S \rightarrow \mathcal{D}$ be a l.s.c. set-valued map with closed decomposable values and let $f(\cdot): S \rightarrow L^{1}\left(\Pi, \mathbb{R}^{n}\right), q(\cdot): S \rightarrow L^{1}(\Pi, \mathbb{R})$ be continuous such that the multifunction $H(\cdot): S \rightarrow \mathcal{D}$ defined by

$$
H(s)=\operatorname{cl}\{v(\cdot) \in G(s) ;\|v(x, y, z)-f(s)(x, y, z)\|<q(s)(x, y, z) \text { a.e. }(\Pi)\}
$$

has nonempty values.

Then $H(\cdot)$ has a continuous selection, i.e. there exists a continuous mapping $h(\cdot): S \rightarrow L^{1}\left(\Pi, \mathbb{R}^{n}\right)$ such that $h(s) \in H(s) \forall s \in S$.

In what follows, by $\Lambda$ we mean the linear subspace of $C\left(\Pi, \mathbb{R}^{n}\right)$ consisting of all $\lambda \in$ $C\left(\Pi, \mathbb{R}^{n}\right)$ such that there exist continuous functions $\varphi: \Pi_{1} \rightarrow \mathbb{R}^{n}, \psi: \Pi_{2} \rightarrow \mathbb{R}^{n}, \chi: \Pi_{3} \rightarrow$ $\mathbb{R}^{n}$ satisfying (1.3) with $\lambda(x, y, z)=\varphi(x, y)+\psi(y, z)+\chi(x, z)-\varphi(x, 0)-\varphi(0, y)-\psi(0, z)+$ $\psi(0,0)=\varphi(x, y)+\psi(y, z)+\chi(x, z)-v_{1}(x)-v_{2}(y)-v_{3}(z)+v_{0},(x, y, z) \in \Pi$. Note that $\Lambda$, equipped with the norm of $C\left(\Pi, \mathbb{R}^{n}\right)$, is a separable Banach space.

For $\sigma \in L^{1}\left(\Pi, \mathbb{R}^{n}\right)$, consider the following Darboux problem

$$
\begin{gathered}
u_{x y z}(x, y, z)=\sigma(x, y, z), \\
u(x, y, 0)=\varphi(x, y), \\
u(0, y, z)=\psi(y, z), \\
u(x, 0, z)=\chi(x, z) .
\end{gathered}
$$

Definition 2.4. Let $\lambda \in \Lambda$. The function $u \in C\left(\Pi, \mathbb{R}^{n}\right)$ given by

$$
u(x, y, z)=\lambda(x, y, z)+\int_{0}^{x} \int_{0}^{y} \int_{0}^{z} \sigma(\xi, \eta, \mu) d \xi d \eta d \mu, \quad(x, y, z) \in \Pi
$$

is said to be a solution of (2.1)

Obviously, problem (2.1) has a unique solution, which will be denoted by $u^{\lambda, \sigma}$.

Definition 2.5. A function $u \in C\left(\Pi, \mathbb{R}^{n}\right)$ is said to be a solution of problem (1.1)-(1.2) if there exists a function $\sigma \in L^{1}\left(\Pi, \mathbb{R}^{n}\right)$ such that

$$
\begin{aligned}
& \sigma(x, y, z) \in F(x, y, z, u(x, y, z)) \text { a.e. (П), } \\
& u(x, y, z)=\lambda(x, y, z)+\int_{0}^{x} \int_{0}^{y} \int_{0}^{z} \sigma(\xi, \eta, \mu) d \xi d \eta d \mu, \quad(x, y, z) \in \Pi .
\end{aligned}
$$

We denote by $\mathcal{S}(\lambda)$ the solution set of (1.1)-(1.2). 


\section{The main results}

In order to obtain a Filippov type existence result for problem (1.1)-(1.2) one needs the following assumptions on $F$.

Hypothesis H1. Let $F: \Pi \times \mathbb{R}^{n} \rightarrow \mathcal{P}\left(\mathbb{R}^{n}\right)$ be a set-valued map with nonempty compact values, satisfying the following assumptions

i) The set-valued maps $(x, y, z) \rightarrow F(x, y, z, u)$ is measurable for all $u \in \mathbb{R}^{n}$.

ii) There exist $k>0$ such that, for almost all $(x, y, z) \in \Pi F(x, y, z, \cdot)$ is $k$-Lipschitz, i.e.

$$
d_{H}\left(F(x, y, z, u), F\left(x, y, z, u^{\prime}\right)\right) \leq k\left\|u-u^{\prime}\right\| \quad \forall u, u^{\prime} \in \mathbb{R}^{n} .
$$

In what follows, $\lambda_{1} \in \Lambda, w \in C\left(\Pi, \mathbb{R}^{n}\right)$ with $w_{x y z} \in L^{1}\left(\Pi, \mathbb{R}^{n}\right), w(x, y, 0)=\lambda_{1}(x, y, 0)$, $w(0, y, z)=\lambda_{1}(0, y, z), w(x, 0, z)=\lambda_{1}(x, 0, z)$ and there exists $q \in L^{1}\left(\Pi, \mathbb{R}_{+}\right)$which satisfies

$$
d\left(w_{x y z}(x, y, z), F(x, y, z, w(x, y, z))\right) \leq q(x, y, z) \text { a.e. }(\Pi) .
$$

Theorem 3.1. Let Hypothesis $H 1$ be satisfied, consider $\lambda \in \Lambda$ and $\lambda_{1} \in \Lambda, w \in C\left(\Pi, \mathbb{R}^{n}\right), q \in$ $L^{1}\left(\Pi, \mathbb{R}_{+}\right)$as above.

If $k T_{1} T_{2} T_{3}<1$ there exists $u$ a solution of problem (1.1)-(1.2) such that

$$
\|u(x, y, z)-w(x, y, z)\| \leq \frac{\left\|\lambda-\lambda_{1}\right\|_{C}+|q|_{1}}{1-k T_{1} T_{2} T_{3}}, \quad \forall(x, y, z) \in \Pi .
$$

Proof. We define $f_{0}=w_{x y z}, u_{0}=w$ and $T=T_{1} T_{2} T_{3}$. It follows from Lemma 2.1 and Hypothesis $\mathrm{H} 1$ that there exists a measurable function $f_{1}$ such that $f_{1}(x, y, z) \in F\left(x, y, z, u_{0}(x, y, z)\right)$ a.e. (П) and for almost all $(x, y, z) \in \Pi$

$$
\left\|f_{0}(x, y, z)-f_{1}(x, y, z)\right\|=d\left(f_{0}(x, y, z), F\left(x, y, z, u_{0}(x, y, z)\right)\right) \leq q(x, y, z) .
$$

Define, for $(x, y, z) \in \Pi$

$$
u_{1}(x, y, z)=\lambda(x, y, z)+\int_{0}^{x} \int_{0}^{y} \int_{0}^{z} f_{1}(r, s, t) d r d s d t
$$

Since

$$
w(x, y, z)=\lambda_{1}(x, y, z)+\int_{0}^{x} \int_{0}^{y} \int_{0}^{z} f_{0}(r, s, t) d r d s d t
$$

one has

$$
\begin{aligned}
\left\|u_{1}(x, y, z)-u_{0}(x, y, z)\right\| & \leq\left\|\lambda(x, y, z)-\lambda_{1}(x, y, z)\right\|+\int_{0}^{x} \int_{0}^{y} \int_{0}^{z}\left\|f_{1}(r, s, t)-f_{0}(r, s, t)\right\| d r d s d t \\
& \leq\left\|\lambda-\lambda_{1}\right\|_{C}+|q|_{1} .
\end{aligned}
$$

From Lemma 2.1 and Hypothesis H1 we deduce the existence of a measurable function $f_{2}$ such that $f_{2}(x, y, z) \in F\left(x, y, z, u_{1}(x, y, z)\right)$ a.e. $(\Pi)$ and for almost all $(x, y, z) \in \Pi$

$$
\begin{aligned}
\left\|f_{2}(x, y, z)-f_{1}(x, y, z)\right\| & \leq d\left(f_{1}(x, y, z), F\left(x, y, z, u_{1}(x, y, z)\right)\right) \leq d_{H}\left(F\left(x, y, z, u_{0}(x, y, z)\right),\right. \\
\left.F\left(x, y, z, u_{1}(x, y, z)\right)\right) & \leq k\left\|u_{1}(x, y, z)-u_{0}(x, y, z)\right\| \leq k\left(\left\|\lambda-\lambda_{1}\right\|_{C}+|q|_{1}\right) .
\end{aligned}
$$


Define, for $(x, y, z) \in \Pi$

$$
u_{2}(x, y, z)=\lambda(x, y, z)+\int_{0}^{x} \int_{0}^{y} \int_{0}^{z} f_{1}(r, s, t) d r d s d t
$$

and one has

$$
\left\|u_{2}(x, y, z)-u_{1}(x, y, z)\right\| \leq \int_{0}^{x} \int_{0}^{y} \int_{0}^{z}\left\|f_{2}(r, s, t)-f_{1}(r, s, t)\right\| d r d s d t \leq k T\left(\left\|\lambda-\lambda_{1}\right\|_{C}+|q|_{1}\right)
$$

Assuming that for some $p \geq 2$ we have already constructed the sequences $\left(u_{i}\right)_{i=1}^{p},\left(f_{i}\right)_{i=1}^{p}$ satisfying

$$
\begin{array}{cl}
\left\|u_{p}(x, y, z)-u_{p-1}(x, y, z)\right\| \leq(k T)^{p-1}\left(\left\|\lambda-\lambda_{1}\right\|_{C}+|q|_{1}\right) & (x, y, z) \in \Pi, \\
\left\|f_{p}(x, y, z)-f_{p-1}(x, y, z)\right\| \leq k(k T)^{p-1}\left(\left\|\lambda-\lambda_{1}\right\|_{C}+|q|_{1}\right) & \text { a.e. (П). }
\end{array}
$$

We apply Lemma 2.1 and we find a measurable function $f_{p+1}$ such that $f_{p+1}(x, y, z) \in$ $F\left(x, y, z, u_{p}(x, y, z)\right)$ a.e. $(\Pi)$ and for almost all $(x, y, z) \in \Pi$

$$
\begin{aligned}
\left\|f_{p+1}(x, y, z)-f_{p}(x, y, z)\right\| & \leq d\left(f_{p+1}(x, y, z), F\left(x, y, z, u_{p}(x, y, z)\right)\right) \leq d_{H}\left(F\left(x, y, z, u_{p}(x, y, z)\right),\right. \\
\left.F\left(x, y, z, u_{p-1}(x, y, z)\right)\right) & \leq k\left\|u_{p}(x, y, z)-u_{p-1}(x, y, z)\right\| \leq k(k T)^{p-1}\left(\left\|\lambda-\lambda_{1}\right\|_{C}+|q|_{1}\right) .
\end{aligned}
$$

Define, for $(x, y, z) \in \Pi$

$$
u_{p+1}(x, y, z)=\lambda(x, y, z)+\int_{0}^{x} \int_{0}^{y} \int_{0}^{z} f_{p+1}(r, s, t) d r d s d t .
$$

We have

$$
\begin{aligned}
\left\|u_{p+1}(x, y, z)-u_{p}(x, y, z)\right\| & \leq \int_{0}^{x} \int_{0}^{y} \int_{0}^{z}\left\|f_{p+1}(r, s, t)-f_{p}(r, s, t)\right\| d r d s d t \\
& \leq \int_{0}^{x} \int_{0}^{y} \int_{0}^{z} k\left\|u_{p}(r, s, t)-u_{p-1}(r, s, t)\right\| d r d s d t \\
& \leq \int_{0}^{x} \int_{0}^{y} \int_{0}^{z} k(k T)^{p-1}\left(\left\|\lambda-\lambda_{1}\right\|_{C}+|q|_{1}\right) d r d s d t \\
& \leq(k T)^{p}\left(\left\|\lambda-\lambda_{1}\right\|_{C}+|q|_{1}\right) .
\end{aligned}
$$

Therefore from (3.2) it follows that the sequence $\left(u_{p}\right)_{p \geq 0}$ is a Cauchy sequence in the space $C\left(\Pi, \mathbb{R}^{n}\right)$, so it converges to $u \in C\left(\Pi, \mathbb{R}^{n}\right)$. From (3.3) it follows that the sequence $\left(f_{p}\right)_{p \geq 0}$ is a Cauchy sequence in the space $L^{1}\left(\Pi, \mathbb{R}^{n}\right)$, thus it converges to $f \in L^{1}\left(\Pi, \mathbb{R}^{n}\right)$.

Using the fact that the values of $F$ are closed we get that $f(x, y, z) \in F(x, y, z, u(x, y, z))$ a.e. (П).

Since

$$
\begin{aligned}
\| \int_{0}^{x} & \int_{0}^{y} \int_{0}^{z} f_{p}(r, s, t) d s d t-\int_{0}^{x} \int_{0}^{y} \int_{0}^{z} f(r, s, t) d r d s d t \| \\
& \leq \int_{0}^{x} \int_{0}^{y} \int_{0}^{z}\left\|f_{p}(r, s, t)-f(r, s, t)\right\| d r d s d t \\
& \leq \int_{0}^{x} \int_{0}^{y} \int_{0}^{z} k\left\|u_{p-1}(r, s, t)-u(r, s, t)\right\| d r d s d t \\
& \leq k T\left\|u_{p-1}-u\right\|_{C},
\end{aligned}
$$


therefore, we may pass to the limit in (3.4) and we obtain that $u(\cdot, \cdot)$ is a solution of problem (1.1)-(1.2). On the other hand, by adding inequalities (3.2) for any $(x, y, z) \in \Pi$ we have

$$
\begin{aligned}
&\left\|u_{p}(x, y, z)-w(x, y, z)\right\| \\
& \leq\left\|u_{p}(x, y, z)-u_{p-1}(x, y, z)\right\| \\
& \quad+\left\|u_{p-1}(x, y, z)-u_{p-2}(x, y, z)\right\|+\cdots+\left\|u_{2}(x, y, z)-u_{1}(x, y, z)\right\| \\
& \quad+\left\|u_{1}(x, y, z)-u_{0}(x, y, z)\right\| \\
& \leq\left((k T)^{p-1}+(k T)^{p-2}+\cdots+k T+1\right)\left(\left\|\lambda-\lambda_{1}\right\|_{C}+|q|_{1}\right) \\
& \leq \frac{\left\|\lambda-\lambda_{1}\right\|_{C}+|q|_{1}}{1-k T} .
\end{aligned}
$$

It remains to pass to the limit with $p \rightarrow \infty$ in (3.5) in order to obtain (3.1) and the proof is complete.

If in Theorem 3.1 we take $w=0, \lambda_{1}=0$ and $q \equiv k$ then we obtain the following existence result for solutions of problem (1.1)-(1.2).

Corollary 3.2. Let Hypothesis $H 1$ be satisfied, $k T_{1} T_{2} T_{3}<1$ and assume that $d(0, F(x, y, z, 0)) \leq k$ $\forall(x, y, z) \in \Pi$.

Then there exists $u \in C\left(\Pi, \mathbb{R}^{n}\right)$ a solution of problem (1.1)-(1.2) such that

$$
\|u(x, y, z)\| \leq \frac{\|\lambda\|_{C}+k T_{1} T_{2} T_{3}}{1-k T_{1} T_{2} T_{3}}, \quad \forall(x, y, z) \in \Pi
$$

We note that the proof of corollary above can be performed also by using the CovitzNadler set-valued contraction principle.

Next we obtain a continuous version of Theorem 3.1. This result allows to provide a continuous selection of the solution set of problem (1.1)-(1.2).

\section{Hypothesis H2.}

i) $S$ is a separable metric space, $\lambda: S \rightarrow \Lambda$ and $\varepsilon(\cdot): S \rightarrow(0, \infty)$ are continuous mappings.

ii) There exist the continuous mappings $\lambda_{1}(\cdot): S \rightarrow \Lambda, g(\cdot): S \rightarrow L^{1}\left(\Pi, \mathbb{R}^{n}\right), w(\cdot): S \rightarrow$ $C\left(\Pi, \mathbb{R}^{n}\right)$ and $q(\cdot): S \rightarrow L^{1}\left(\Pi, \mathbb{R}_{+}\right)$such that $w(s)_{x y z} \equiv g(s), w(s)(x, y, 0)=\lambda_{1}(s)(x, y, 0)$, $w(s)(0, y, z)=\lambda_{1}(s)(0, y, z), w(s)(x, 0, z)=\lambda_{1}(s)(x, 0, z)(x, y, z) \in \Pi, s \in S$ and

$$
d(g(s)(x, y, z), F(x, y, z, w(s)(x, y, z)) \leq q(s)(x, y, z) \quad \text { a.e. }(\Pi), \forall s \in S .
$$

Theorem 3.3. Assume that Hypotheses $\mathrm{H} 1$ and $\mathrm{H} 2$ are satisfied.

Then, there exists a continuous mapping $u(\cdot): S \rightarrow C\left(\Pi, \mathbb{R}^{n}\right)$ such that for any $s \in S, u(s)$ is a solution of problem (1.1) which satisfies $u(s)(x, y, 0)=\lambda(s)(x, y, 0), u(s)(0, y, z)=\lambda(s)(0, y, z)$, $u(s)(x, 0, z)=\lambda(s)(x, 0, z),(x, y, z) \in \Pi, s \in S$ and, for any $(x, y, z) \in \Pi, s \in S$,

$$
\|u(s)(x, y, z)-w(s)(x, y, z)\| \leq \frac{\left\|\lambda(s)-\lambda_{1}(s)\right\|_{C}+\varepsilon(s)+\|q(s)\|_{1}}{1-k T_{1} T_{2} T_{3}} .
$$

Proof. We make the following notations $u_{0}=w, k_{p}(s):=(k T)^{p-1}\left(\left\|\lambda(s)-\lambda_{1}(s)\right\|_{C}+\varepsilon(s)+\right.$ $\left.\|q(s)\|_{1}\right), p \geq 1, T=T_{1} T_{2} T_{3}$. 
We consider the set-valued maps $G_{0}, H_{0}$ defined, respectively, by

$$
\begin{aligned}
& G_{0}(s)=\left\{v \in L^{1}\left(\Pi, \mathbb{R}^{n}\right) ; v(x, y, z) \in F(x, y, z, w(s)(x, y, z)) \text { a.e. }(\Pi)\right\}, \\
& H_{0}(s)=\operatorname{cl}\left\{v \in G_{0}(s) ;\|v(x, y, z)-g(s)(x, y, z)\|<q(s)(x, y, z)+\frac{\varepsilon(s)}{T}\right\} .
\end{aligned}
$$

Since $d\left(g(s)(x, y, z), F(x, y, z, w(s)(x, y, z)) \leq q(s)(x, y, z)<q(s)(x, y, z)+\frac{\varepsilon(s)}{T}\right.$ the set $H_{0}(s)$ is not empty.

Set $F_{0}^{*}(x, y, z, s)=F(x, y, z, w(s)(x, y, z))$ and note that

$$
d\left(0, F_{0}^{*}(x, y, z, s)\right) \leq\|g(s)(x, y, z)\|+q(s)(x, y, z)=: q^{*}(s)(x, y, z)
$$

and $q^{*}(\cdot): S \rightarrow L^{1}(I, \mathbb{R})$ is continuous.

Applying now Lemmas 2.2 and 2.3 we obtain the existence of a continuous selection $f_{0}$ of $H_{0}$ such that $\forall s \in S,(x, y, z) \in \Pi$,

$$
\begin{gathered}
f_{0}(s)(x, y, z) \in F(x, y, z, w(s)(x, y, z)) \quad \text { a.e. }(\Pi), \forall s \in S \\
\left\|f_{0}(s)(x, y, z)-g(s)(x, y, z)\right\| \leq q_{0}(s)(x, y, z)=q(s)(x, y, z)+\frac{\varepsilon(s)}{T} .
\end{gathered}
$$

We define

$$
u_{1}(s)(x, y, z)=\lambda(s)(x, y, z)+\int_{0}^{x} \int_{0}^{y} \int_{0}^{z} f_{0}(s)(r, l, t) d r d l d t
$$

and one has

$$
\begin{aligned}
\| u_{1}(s) & (x, y, z)-u_{0}(s)(x, y, z) \| \\
\leq & \left\|\lambda(s)-\lambda_{1}(s)\right\|_{C}+\int_{0}^{x} \int_{0}^{y} \int_{0}^{z}\left\|f_{0}(s)(r, l, t)-g(s)(r, l, t)\right\| d r d l d t \\
\leq & \left\|\lambda(s)-\lambda_{1}(s)\right\|_{C}+\int_{0}^{x} \int_{0}^{y} \int_{0}^{z}\left[q(s)(r, l, t)+\frac{\varepsilon(s)}{T}\right] d r d l d t \\
& =\left\|\lambda(s)-\lambda_{1}(s)\right\|_{C}+\|q(s)\|_{1}+\varepsilon(s)=k_{1}(s)
\end{aligned}
$$

We shall construct, using the same idea as in [6], two sequences of approximations $f_{p}$ : $S \rightarrow L^{1}\left(\Pi, \mathbb{R}^{n}\right), u_{p}: S \rightarrow C\left(\Pi, \mathbb{R}^{n}\right)$ with the following properties

a) $f_{p}: S \rightarrow L^{1}\left(\Pi, \mathbb{R}^{n}\right), u_{p}: S \rightarrow C\left(\Pi, \mathbb{R}^{n}\right)$ are continuous,

b) $f_{p}(s)(x, y, z) \in F\left(x, y, z, u_{p}(s)(x, y, z)\right)$, a.e. $(\Pi), s \in S$,

c) $\left\|f_{p}(s)(x, y, z)-f_{p-1}(s)(x, y, z)\right\| \leq k \cdot k_{p}(s)$, a.e. $(\Pi), s \in S$,

d) $u_{p+1}(s)(x, y, z)=\lambda(s)(x, y, z)+\int_{0}^{x} \int_{0}^{y} \int_{0}^{z} f_{p}(s)(r, l, t) d r d l d t$.

Suppose we have already constructed $f_{i}, u_{i}$ satisfying a) $-\mathrm{c}$ ) and define $u_{p+1}$ as in d). From c) and d) one has

$$
\begin{aligned}
\| u_{p+1} & (s)(x, y, z)-u_{p}(s)(x, y, z) \| \\
\leq & \int_{0}^{x} \int_{0}^{y} \int_{0}^{z}\left\|f_{p}(s)(r, l, t)-f_{p-1}(s)(r, l, t)\right\| d r d l d t \\
\leq & \int_{0}^{x} \int_{0}^{y} \int_{0}^{z} k \cdot k_{p}(s) d r d l d t=(k T) k_{p}(s)=k_{p+1}(s)
\end{aligned}
$$


On the other hand,

$$
\begin{aligned}
d\left(f_{p}(s)(x, y, z), F\left(x, y, z, u_{p+1}(s)(x, y, z)\right)\right. & \\
& \leq k\left\|u_{p+1}(s)(x, y, z)-u_{p}(s)(x, y, z)\right\|<k \cdot k_{p+1}(s) .
\end{aligned}
$$

For any $s \in S$ we define the set-valued maps

$$
\begin{aligned}
& G_{p+1}(s)=\left\{v \in L^{1}\left(\Pi, \mathbb{R}^{n}\right) ; v(x, y, z) \in F\left(x, y, z, u_{p+1}(s)(x, y, z)\right) \text { a.e. }(\Pi)\right\}, \\
& H_{p+1}(s)=\operatorname{cl}\left\{v \in G_{p+1}(s) ;\left\|v(x, y, z)-f_{p}(s)(x, y, z)\right\|<k \cdot k_{p+1}(s)\right\} .
\end{aligned}
$$

We note that from (3.7) the set $H_{p+1}(s)$ is not empty.

Set $F_{p+1}^{*}(x, y, z, s)=F\left(x, y, z, u_{p+1}(s)(x, y, z)\right)$ and note that

$$
d\left(0, F_{p+1}^{*}(x, y, z, s)\right) \leq\left\|f_{p}(s)(x, y, z)\right\|+k \cdot k_{p+1}(s)=: q_{p+1}^{*}(s)(x, y, z)
$$

and $q_{p+1}^{*}(\cdot): S \rightarrow L^{1}(I, \mathbb{R})$ is continuous.

By Lemmas 2.2 and 2.3 we obtain the existence of a continuous function $f_{p+1}: S \rightarrow$ $L^{1}\left(\Pi, \mathbb{R}^{n}\right)$ such that

$$
\begin{array}{cl}
f_{p+1}(s)(x, y, z) \in F\left(x, y, z, u_{p+1}(s)(x, y, z)\right) & \text { a.e. }(\Pi), \forall s \in S, \\
\left\|f_{p+1}(s)(x, y, z)-f_{p}(s)(x, y, z)\right\| \leq k \cdot k_{p+1}(s) & \forall s \in S,(x, y, z) \in \Pi .
\end{array}
$$

From (3.6), c) and d) we obtain

$$
\begin{aligned}
\left\|u_{p+1}(s)-u_{p}(s)\right\|_{C} & \leq k_{p+1}(s)=(k T)^{p}\left(\left\|\lambda(s)-\lambda_{1}(s)\right\|_{C}+\varepsilon(s)+\|q(s)\|_{1}\right), \\
\left\|f_{p+1}(s)-f_{p}(s)\right\|_{1} & \leq k T \cdot k_{p}(s)=(k T)^{p}\left(\left\|\lambda(s)-\lambda_{1}(s)\right\|_{C}+\varepsilon(s)+\|q(s)\|_{1}\right) .
\end{aligned}
$$

Therefore $f_{p}(s), u_{p}(s)$ are Cauchy sequences in the Banach space $L^{1}\left(\Pi, \mathbb{R}^{n}\right)$ and $C\left(\Pi, \mathbb{R}^{n}\right)$, respectively. Let $f: S \rightarrow L^{1}\left(\Pi, \mathbb{R}^{n}\right), x: S \rightarrow C\left(\Pi, \mathbb{R}^{n}\right)$ be their limits. The function $s \rightarrow$ $\left\|\lambda(s)-\lambda_{1}(s)\right\|_{C}+\varepsilon(s)+\|q(s)\|_{1}$ is continuous, hence locally bounded. Therefore (3.9) implies that for every $s^{\prime} \in S$ the sequence $f_{p}\left(s^{\prime}\right)$ satisfies the Cauchy condition uniformly with respect to $s^{\prime}$ on some neighborhood of $s$. Hence, $s \rightarrow f(s)$ is continuous from $S$ into $L^{1}\left(\Pi, \mathbb{R}^{n}\right)$.

From (3.8), as before, $u_{p}(s)$ is Cauchy in $C\left(\Pi, \mathbb{R}^{n}\right)$ locally uniformly with respect to $s$. So, $s \rightarrow u(s)$ is continuous from $S$ into $C\left(\Pi, \mathbb{R}^{n}\right)$. On the other hand, since $u_{p}(s)$ converges uniformly to $u(s)$ and a.e. ( $\Pi)$ and $\forall s \in S$

$$
d\left(f_{p}(s)(x, y, z), F(x, y, z, u(s)(x, y, z)) \leq k\left\|u_{p}(s)(x, y, z)-u(s)(x, y, z)\right\|,\right.
$$

passing to the limit along a subsequence of $f_{p}(s)$ converging pointwise to $f(s)$ we obtain

$$
f(s)(x, y, z) \in F(x, y, z, u(s)(x, y, z)) \text { a.e. }(\Pi), \forall s \in S .
$$

One the other hand,

$$
\begin{aligned}
\| \int_{0}^{x} & \int_{0}^{y} \int_{0}^{z} f_{p}(s)(r, l, t) d r d l d t-\int_{0}^{x} \int_{0}^{y} \int_{0}^{z} f(s)(r, l, t) d r d l d t \| \\
& \leq \int_{0}^{x} \int_{0}^{y} \int_{0}^{z}\left\|f_{p}(s)(r, l, t)-f(s)(r, l, t)\right\| d r d l d t \leq k T\left\|u_{p-1}(s)-u(s)\right\|_{C} .
\end{aligned}
$$

Therefore one may pass to the limit in d) and we get $\forall(x, y, z) \in \Pi, s \in S$

$$
u(s)(x, y, z)=\lambda(s)(x, y, z)+\int_{0}^{x} \int_{0}^{y} \int_{0}^{z} f(s)(r, l, t) d r d l d t,
$$


i.e., $u(s)$ is the desired solution.

Moreover, by adding inequalities (3.6) for all $p \geq 1$ we get

$$
\left\|u_{p+1}(s)(x, y, z)-w(s)(x, y, z)\right\| \leq \sum_{l=1}^{p+1} k_{l}(s) \leq \frac{\left\|\lambda(s)-\lambda_{1}(s)\right\|_{C}+\varepsilon(s)+\|q(s)\|_{1}}{1-k T} .
$$

Passing to the limit in (3.10) we obtain the conclusion of the theorem.

Corollary 3.4. Assume that Hypothesis $H 2$ is satisfied and, in addition, $d(0, F(x, y, z, 0)) \leq k$ a.e. (П).

Then, there exists a function $u(\cdot, \cdot): \Pi \times \Lambda \rightarrow \mathbb{R}^{n}$ such that

a) $x(\cdot, \lambda) \in \mathcal{S}(\lambda), \forall \lambda \in \Lambda$.

b) $\lambda \rightarrow x(\cdot, \lambda)$ is continuous from $\Lambda$ into $C\left(\Pi, \mathbb{R}^{n}\right)$.

Proof. We take $S=\Lambda, \varepsilon$ an arbitrary continuous positive function, $g(\cdot)=0, w(\cdot)=0$, $q(s)(x, y, z) \equiv k$ and we apply Theorem 3.3 in order to obtain the conclusion of the corollary.

\section{Acknowledgements}

Work supported by the CNCS grant PN-II-ID-PCE-2011-3-0198

The author would like to thank to the anonymous referee for his helpful comments which improved the presentation of the paper.

\section{References}

[1] D. Azzam-Laouir, F. Bounama, Second-order differential inclusions with Lipschitz right-hand side, Electron. J. Differential Equations 2010, No. 85, 1-9. MR2680288

[2] A. Bressan, G. Colombo, Extensions and selections of maps with decomposable values, Studia Math. 90(1988), 69-86. MR0947921

[3] C. Castaing, M. Valadier, Convex analysis and measurable multifunctions, Springer, Berlin, 1977. MR0467310

[4] K. Deimling, Das charakteristische Anfangswertproblem für $u_{x_{1} x_{2} x_{3}}=f$ unter Carathéodory-Voraussetzungen (in German), Arch. Math. (Basel) 22(1971), 514-522. MR0310443

[5] A. F. Filippov, Classical solutions of differential equations with multi-valued right-hand side, SIAM J. Control 5(1967), 609-621. MR0220995

[6] V. Staicu, On a nonconvex hyperbolic differential inclusion, Proc. Edinburgh Math. Soc. (2) 35(1992), 375-382. MR1878653

[7] G. Teodonu, The Darboux problem for third order hyperbolic inclusions, Libertas Math. 23(2003), 119-127. MR2002312 
[8] G. Teodonu, The Darboux problem for third order hyperbolic inclusions via continuous selections for continuous multifunctions, Fixed Point Theory 11(2010), 133-142. MR2656015

[9] G. Teodoru, The Darboux problem for third order hyperbolic inclusions via contraction principle of Covitz and Nadler, in: Proceedings of $10^{\text {th }}$ IC-FPTA, Editors: R. Espinola, A. Petrușel, S. Prus, 2011, pp. 241-252. MR3203588

[10] H. D. Tuan, On local controllability of hyperbolic inclusions, J. Math. Systems Estim. Control 4(1994), 319-339. MR1298839

[11] H. D. TuAn, On solution sets of nonconvex Darboux problems and applications to optimal control with end point constraints, J. Austral. Math. Soc. Ser. B 37(1996), 354-391. MR1376040 